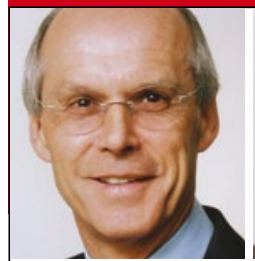

Prof. Dr. med. H. S. FüeßI Isar-AmperKlinikum, $\begin{array}{ll}\text { Klinikum, } & \text { Kardiologische } \\ \text { KL. München-Ost } & \text { Praxis, München }\end{array}$ Haar

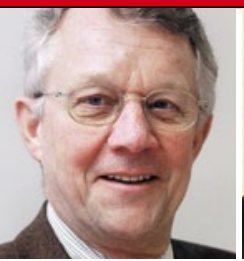

Prof. Dr. med. H. Holzgreve Internist, Praxis, München

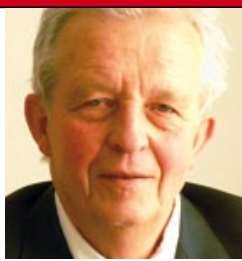

Prof. Dr. med. K. Malberg Immunologie, DresdenLoschwitz

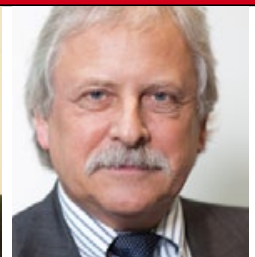

Prof. Dr. med. H.-C. Diener

Klinik für Neurologie, Universitätsklinik Essen

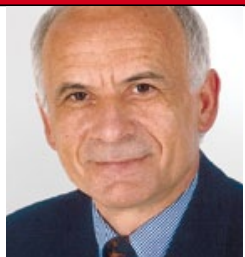

Prof. Dr. med.

A. Wirth

Teutoburger-

Wald-Klinik,

Bad Rothenfelde

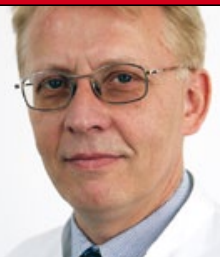

Prof. Dr. med.

K. Rasche

HELIOS Klinikum

Wuppertal

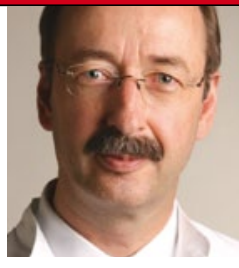

Prof. Dr. med J. Labenz

Ev. Jung-StillingKrankenhaus Siegen

\title{
Herzinfarktsymptome und ihre prognostische Bedeutung
}

\begin{abstract}
Die subjektiven Beschwerden bei akutem Myokardinfarkt sind prognostisch bedeutsam: Todesangst, Schwitzen und Schmerzen im oberen Abdomen signalisieren einen eher guten, Synkopen und Atemnot einen schlechten Verlauf.
\end{abstract}

- Die Autoren des MONICA/KORAProjekts in der Region Augsburg haben 13 Symptome (Schmerzen an sechs

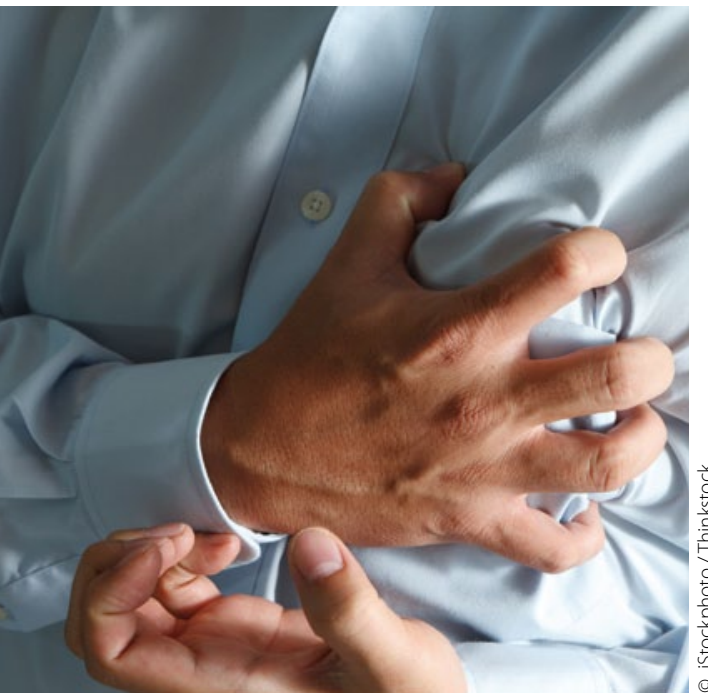

Schwere Symptome - bessere Prognose.
Körperstellen, Todesangst, Schwindel, Schwitzen, Übelkeit, Atemnot, Erbrechen und Synkope) beim ersten Herzinfarkt von 1231 Männern und 415 Frauen im Alter von 25 bis 74 Jahren sorgfältig registriert und mit dem weiteren Verlauf korreliert. Bei der Auswertung wurden Einflussfaktoren wie Alter, Geschlecht, Infarkt-Typ, Reperfusionstherapie, Diabetes mellitus und Dauer bis zur stationären Aufnahme berücksichtigt.

Die Mortalität innerhalb der ersten vier Wochen lag bei $6,1 \%$ und nach im Mittel 4,1 Jahren bei 10,6\%. Unter den 13 Symptomen waren kurzfristig Todesangst (-89\%) und Schwitzen (-55\%) mit einer guten, Synkopen mit einer 5,3-fach schlechteren Prognose belastet. Anderseits waren langfristig Schmerzen im oberen Abdomen und Schmerzen bzw. Druckgefühl in der Brust gute, Atemnot (1,5-fach) schlechte Vorzeichen. Asymptomatische Infarkte waren mit einer um das 1,85-fach erhöhten Mortalität assoziiert.

\section{- I. Kirchberger et al.}

Presenting symptoms of myocardial infarction predict short- and long-term mortality: The MONICA/KORA Myocardial Infarction Registry. A. Heart J. 164 (2012) 856-861

\section{Kommentar}

Wichtig ist zunächst einmal, dass Symptome des akuten Infarkts den Patienten alarmieren und möglichst schnell in ärztliche Behandlung und Überwachung führen, denn asymptomatische Infarkte haben die höchste Langzeitmortalität. Beim Myokardinfarkt denkt jeder, ob Arzt oder Laie, zuerst an die klassischen Symptome Schmerzen, Druck- oder Engegefühl über der Brust, an Hals, Schulter oder Arm. Diese Symptome sind in der vorliegenden Untersuchung mit einer geringeren Mortalität verknüpft. Wahrscheinlich nicht, weil sie eine bessere Prognose anzeigen, sondern weil jeder sie als Alarmsymptome kennt und schnell den Arzt oder die Notaufnahme aufsucht. Als Konsequenz aus dieser Untersuchung müsste man die sogenannten atypischen Beschwerden aufwerten, damit Laien auch dann an einen Infarkt denken und Ärzte nicht erst auf diagnostischen Umwegen zur richtigen Diagnose gelangen. Doch bleibt das breite Spektrum subjektiver Beschwerden bei Herzinfarkt problematisch. Denn das für den Patienten schlimmste Symptom, die Todesangst, signalisiert nicht die Schwere des Infarktes, sondern eine individuelle, emotionale Reaktion bei typischen oder auch atypischen Beschwerden.

H. HOLZGREVE = 